\title{
Insatisfação corporal em universitários de diferentes áreas de conhecimento
}

\author{
Body dissatisfaction in college students of different study areas
}

Valter Paulo Neves Miranda', Juliana Fernandes Filgueiras', Clara Mockdece Neves', Paula Costa Teixeira², Maria Elisa Caputo Ferreira'

\section{RESUMO}

Objetivos: Verificar a prevalência de insatisfação corporal em universitários de diferentes áreas de conhecimento, bem como a relação com sexo e com estado nutricional. Métodos: A amostra foi composta por universitários da Universidade Federal de Juiz de Fora (UFJF) de diferentes áreas de conhecimento. O Body Shape Questionnaire (BSQ) e a Escala de Silhuetas para adultos avaliaram a insatisfação corporal. Massa corporal e estatura autorreferidas foram utilizadas para o cálculo do índice de massa corporal (IMC). Para as análises estatísticas, foram realizados testes de associação, comparação e regressão logística.

Resultados: Dos 535 estudantes, com média de idade de 20,82 \pm 3,03 anos, 245 eram do sexo masculino. A média do BSQ foi de $68,00 \pm 28,74$, sendo $88,9 \%$ livres de insatisfação. Porém, pela escala de silhuetas, $76,6 \%$ foram considerados insatisfeitos. Os estudantes da área de saúde e humanas foram, sem significância, mais insatisfeitos que alunos de exatas.

\section{Palavras-chave}

Imagem corporal, estudantes, estado nutricional, regressão logística.
As mulheres em relação aos homens $(p<0,05)$ e aqueles com sobrepeso/obesidade (OR: 3,$174 ; p=0,000$ ) tiveram a maior frequência na classificação de insatisfação corporal. Conclusão: A maioria dos jovens mostrou-se livre de insatisfação corporal, não havendo relação com a área de estudo, porém as universitárias com IMC mais elevado apresentaram-se mais insatisfeitas com sua imagem corporal.

\section{ABSTRACT}

Objectives: To check the prevalence of body dissatisfaction in college students of different majors, as well as the relationship with gender and nutritional status. Methods: The sample was composed of college students from the Federal University of Juiz de Fora (UFJF) of several different majors. The Body Shape Questionnaire (BSQ) and the Silhouette Scale for Adults assessed body dissatisfaction. Self-referred body mass and height were used to calculate the body mass index (BMI). For the statistical analysis, association, comparison, and logistic regression tests were performed. Results: Among the 535 students, with average age of $20.82 \pm 3.03$ years old, 245 were male. The BSQ average score was 68.00 \pm 28.74 , being $88.9 \%$ of the subjects free from dissatisfaction. Nevertheless, according to the silhouette scale, $76.6 \%$ were found to be dissatisfied. The students of Life Sciences and Humanities were, insignificantly, more dissatisfied than the Exact Sciences students. Women, when compared to men $(p<0.05)$ and those overweight/obese $(O R: 3.174 ; p=0.000)$

1 Universidade Federal de Juiz de Fora (UFJF), Faculdade de Educação Física e Desportos, Conselho Nacional de Desenvolvimento Científico e Tecnológico (CNPq), Grupo de Estudos Corpo e Diversidade Humana.

2 Faculdade de Medicina da Universidade de São Paulo (FMUSP), Instituto de Psiquiatria (IPq), Programa de Transtornos Alimentares (Ambulim).

Endereço para correspondência: Valter Paulo Neves Miranda

Rua Dr. Gil Horta, 163, ap. 402, Centro

36016-400 - Juiz de Fora, MG, Brasil

Telefone: (32) 9928-9878

E-mail:vpnmiranda@yahoo.com.br 


\section{Keywords}

Body image, college

students, nutritional status,

logistic regression. had the highest score in the body dissatisfaction rating. Conclusion: Most young people were found to be free from body dissatisfaction, and no correlation to their area of studies was found. However, the college girls with the highest BMls were found to be the most dissatisfied with their body image.

\section{INTRODUÇÃO}

A imagem corporal (IC) pode ser definida como uma ilustração que se tem na mente acerca do tamanho, da aparência e da forma do corpo, sustentada por dois componentes: o perceptivo, que corresponde às imagens construídas na mente, e o atitudinal, que engloba os pensamentos e sentimentos em relação ao corpo'. A insatisfação corporal é um componente da dimensão atitudinal da IC e pode ser compreendida como uma avaliação negativa que o sujeito faz em relação à sua aparência física². As experiências vivenciadas pelo indivíduo criam um referencial do seu corpo, e essa representação é reconstruída ao longo da vida'.

Um sentimento negativo com a IC na maioria das vezes pode ser desencadeado por meio de dois mecanismos primários: comparação da aparência dos jovens entre si e a internalização de um modelo ideal de magreza ${ }^{3}$. Atualmente, o modelo de beleza imposto pela sociedade corresponde a um corpo magro para as mulheres e musculoso para os homens ${ }^{4}$. Assim, indivíduos que não se encontram nesse ideal de corpo tendem a adotar comportamentos inadequados para o controle de peso, como a procura exacerbada por exercícios físicos, cirurgias plásticas e atitudes alimentares inadequadas ${ }^{5}$.

Geralmente, as pessoas com alta insatisfação com sua imagem corporal podem ficar mais suscetíveis ao desenvolvimento de transtornos alimentares, além de estarem mais sujeitas a buscar dietas inadequadas e abusar do exercício, o que prejudica a qualidade de vida ${ }^{3,5}$.

Algumas pesquisas mostram que os jovens estão cada vez mais vulneráveis a mudanças de comportamento por causa da proximidade com a vida adulta e do ingresso no meio universitário, que proporciona novas relações sociais ${ }^{6}$. Alvarenga et al. ${ }^{7}$ verificaram, em universitárias da área da saúde de diferentes regiões brasileiras, insatisfação corporal bastante expressiva, assim como Costa e Vasconcelos ${ }^{8}$, que encontraram indicadores de elevada rejeição com a forma física em universitários ingressantes de uma instituição pública de Santa Catarina, Brasil.

Outro importante fator a ser considerado são os trabaIhos envolvendo a avaliação da insatisfação corporal que sugerem uma associação significativa com a classificação de sobrepeso e obesidade, a partir do índice de massa corporal $(\mathrm{IMC})^{7,9}$. Atualmente, algumas pesquisas mostram que os sujeitos com excesso de peso são mais suscetíveis à insatisfa- ção com a IC $\mathrm{C}^{10}$. Outros estudos indicam alta prevalência de insatisfação corporal em indivíduos com estado nutricional considerado adequado ${ }^{11}$, principalmente universitários do sexo feminino ${ }^{7,12}$.

São escassos na literatura estudos utilizando instrumentos devidamente adaptados transculturalmente para uma amostra representativa de estudantes universitários brasilei$\operatorname{ros}^{12}$, principalmente pesquisas que avaliam jovens de ambos os gêneros de diferentes áreas de conhecimento.

Assim, esta pesquisa teve como objetivo verificar a prevalência de insatisfação corporal em universitários de diferentes áreas de conhecimento, bem como a relação com o sexo e com o estado nutricional.

\section{MÉTODOS}

Esta pesquisa epidemiológica caracteriza-se como transversal e correlacional ${ }^{13}$. Está de acordo com as normas da Portaria no 196/96, do Conselho Nacional de Saúde, e foi aprovada pelo Comitê de Ética em Pesquisa em Seres Humanos da Universidade Federal de Juiz de Fora (UFJF), sob Parecer nº 333/2009 e Protocolo no 1920.264.2009. A pesquisa foi desenvolvida pelo Laboratório de Estudos do Corpo (LABESC) da Faculdade de Educação Física e Desportos (FAEFID) UFJF. Todos os participantes assinaram o Termo de Consentimento Livre e Esclarecido (TCLE).

\section{Amostra}

Para o cálculo do tamanho da amostra representativa da população, foi utilizada a metodologia recomendada por Thomas et al. ${ }^{13}$. Considerou-se um nível de confiança igual a 95\% e um erro máximo permitido, com precisão absoluta de 5 pontos para mais ou para menos na variável resposta de referência, o escore do Body Shape Questionnaire (BSQ) ${ }^{14}$. Com a maior variabilidade de pontuação verificada para o sexo feminino ( $\pm 31,3$ pontos), como a pior situação ao nível de significância de 5\%, obteve-se um tamanho de amostra mínimo necessário igual a 129, já feita a correção para populações finitas. Com base no cálculo amostral, estimou-se que seria necessário multiplicar por três (número de áreas de conhecimento) o valor amostral mínimo $(n=129)$, obtendo-se um resultado igual a 387 universitários.

A partir de então, buscaram-se informações sobre o número de alunos matriculados na UFJF no ano letivo de 2009, 
destacando a divisão nas três grandes áreas de conhecimento: humanas, saúde e exatas. Nesse período a universidade possuía aproximadamente 10.822 alunos, sendo 34,80\% da área de humanas, 31,60\% da área de saúde e 28,27\% da área de exatas, dados esses divulgados pela própria universidade ${ }^{15}$. Para atender ao valor amostral mínimo $(n=387)$, foram investigados 5\% do número total de alunos (10.822), mantendo a proporcionalidade de cada área do conhecimento. A seleção foi feita de modo aleatório simples nos departamentos das faculdades da UFJF. No total, foram entrevistados 580 universitários, sendo excluídos 55 indivíduos que se recusaram a participar ou não assinaram o TCLE $(n=55)$. Dessa forma, a amostra final foi composta por 535 universitários, sendo 169 da área de exatas, 186 de humanas e 180 da saúde.

\section{Instrumentos}

Para avaliar a insatisfação e as preocupações com a forma do corpo, foi utilizado o BSQ validado para uma população não clínica de universitários brasileiros por Di Pietro e Silveira ${ }^{14}$. O questionário é composto por 34 questões, em escala tipo Likert, em que o avaliado aponta com que frequência, nas últimas quatro semanas, vivenciou os eventos propostos pelas alternativas. As respostas variam de 1 (nunca) a 6 (sempre), sendo a soma das pontuações de cada item o escore final da escala.

A classificação dos resultados do BSQ é dividida em quatro níveis de insatisfação corporal. A pontuação abaixo de 110 indica ausência de insatisfação; entre 111 e 138, insatisfação leve; entre 139 e 167, insatisfação moderada; e pontuação igual ou acima de 168 indica grave insatisfação corporal. Vale ressaltar que essa classificação foi proposta por Cooper et al. ${ }^{16}$, criadores desse instrumento.

Como segundo instrumento avaliativo da insatisfação com a IC, utilizou-se a Escala de Silhuetas Brasileiras para adultos $^{17}$, criada e validada para adultos e crianças brasileiras. Composta por 15 silhuetas, a escala possui inter-relação com o IMC, variando entre 12,5 e $47,5 \mathrm{~kg} / \mathrm{m}^{2}$, com intervalo de 2,5 $\mathrm{kg} / \mathrm{m}^{2}$ entre cada silhueta para ambos os sexos.

O sujeito deveria assinalar "qual a silhueta que melhor representava seu corpo na atualidade" e "qual silhueta ele(a) desejaria ter". Assim, essa escala permite a avaliação do nível de satisfação (NS) calculado a partir da diferença entre a siIhueta ideal (SI) e a silhueta atual (SA). Os valores iguais a zero $(N S=0)$ indicam que os jovens se sentem satisfeitos com sua IC. Já os valores diferentes de zero $(N S \neq 0)$ indicam que o sujeito está insatisfeito com sua IC. Assim, resultados positivos indicam um desejo de aumentar o tamanho corporal, enquanto resultados negativos, um desejo de diminuí-lo ${ }^{18}$.

Para o cálculo do IMC, foram utilizados os dados de massa corporal e estatura autorreferidos. A classificação primária do estado nutricional (EN) foi realizada a partir dos pontos de corte do IMC, estabelecidos pela Organização Mundial da
Saúde ${ }^{19}$ - < 18,5: baixo IMC; entre 18,5 e 24,9: normal IMC; entre 24,9 e 29,9: sobrepeso; > 29,9: obesidade.

\section{Procedimentos}

Inicialmente, os pesquisadores foram até as faculdades e apresentaram o projeto da pesquisa para a coordenação de curso. Em seguida, foi estabelecido contato com o professor para explicar todo o procedimento adotado e pedir autorização para intervenção durante sua aula.

Posteriormente, os pesquisadores realizaram uma explicação sobre os objetivos e procedimentos da pesquisa para os alunos e distribuíram o TCLE. Assim, aqueles que aceitaram participar voluntariamente da pesquisa assinaram o termo e receberam um material de avaliação contendo: cabeçalho para informações gerais (massa corporal, estatura, data de nascimento, sexo e área de conhecimento), questionário BSQ e a Escala de Silhuetas.

Após a conclusão do procedimento de coleta, os dados foram levados, em sigilo total, para o Laboratório de Estudo do Corpo (LABESC), onde foram tabulados e analisados pelos pesquisadores responsáveis pelo estudo.

\section{Análise estatística}

Para análise estatística, utilizou-se o software estatístico SPSS, versão 17.0, com nível de significância de $5 \%(a=0,05)$ para todas as análises. Realizou-se inicialmente análise descritiva de cada variável, por meio da frequência, valores médios e desvios-padrão. Como as variáveis numéricas não apresentaram normalidade pelo teste de Kolmogorov-Smirnov, optou-se pela aplicação de testes não paramétricos.

O teste qui-quadrado foi aplicado para analisar as associações das áreas de conhecimento, o sexo e o estado nutricional em relação à frequência de sujeitos classificados como satisfeitos e insatisfeitos pela Escala de Silhuetas e pelo BSQ.

A variação dos escores da insatisfação com a imagem corporal entre as diferentes áreas de conhecimento e os grupos do estado nutricional foi avaliada pelo teste de Kruskal-Wallis e o teste post hoc de Bonferroni $(a=0,016)$. Para a comparação das médias dos escores dos instrumentos entre ambos os sexos, foi utilizado o teste de Mann-Whitney; para a comparação entre os grupos relacionados ao estado nutricional, utilizou-se o teste qui-quadrado de Pearson de associação linear, e o teste exato de Fisher foi também selecionado para verificar a diferença de categorização de insatisfação corporal entre as variáveis independentes.

Por fim, realizou-se um modelo de regressão logística binária para verificar quais das variáveis independentes analisadas apresentavam associação (risco ou proteção) significativa (p) com a insatisfação corporal (Escala de Silhueta e BSQ), de acordo com a interpretação dos valores do odds ratio (OR). As análises tiveram como base as variáveis que demonstraram relação com a avaliação da insatisfação corporal em outros estudos com universitários brasileiros ${ }^{7,12}$ : os pertencentes da 
área de saúde, o sexo masculino e a classificação IMC normal e baixo. Para atender ao requisito de dicotomia da variável resposta, o BSQ foi reorganizado em duas categorias: satisfeitos - aqueles classificados como livres de insatisfação corporal; e insatisfeitos - aqueles com algum nível de insatisfação corporal (leve, moderada e grave). O mesmo procedimento foi utilizado para a variável estado nutricional, que teve as quatro categorias agrupadas em duas: baixo peso/eutrofia e sobrepeso/obesidade. Esses agrupamentos propiciaram também um número mínimo necessário de elementos em cada categoria de análise.

\section{RESULTADOS}

Participaram da pesquisa 535 estudantes da UFJF no ano de 2010, com média de idade de 20,82 $\pm 3,03$ anos, sendo 245 $(44,2 \%)$ do sexo masculino e $290(55,8 \%)$ do sexo feminino. A média de peso corporal, estatura e IMC autorreferidos foi de $65,69 \pm 14,16 \mathrm{~kg}, 1,70 \pm 0,90 \mathrm{~m}$ e $22,33 \pm 4,06 \mathrm{~kg} / \mathrm{m}^{2}$, respectivamente. A maioria dos jovens apresentou a classificação normal do estado nutricional, sendo $75,2 \%$ da amostra total. Por outro lado, 18,3\% dos universitários apresentaram sobrepeso e obesidade, sendo os estudantes de humanas aqueles com maior frequência de sobrepeso e obesidade, com $18,7 \%$ contra $18,5 \%$ dos estudantes de exatas e $17,8 \%$ dos estudantes da área de saúde.

A média de pontuação do BSQ foi de 68,00 \pm 28,74 pontos. Não houve variação da insatisfação corporal em relação às áreas de conhecimentos pela análise do BSQ $(p=0,513)$, sendo a área de saúde com a maior média com 69,82 $\pm 29,4$ pontos, contra $65,47 \pm 26,35$ e 68,52 \pm 30,04 pontos dos alunos de exatas e humanas, respectivamente. A média dos índices de insatisfação corporal das mulheres foi significativamente maior que dos homens $(p<0,05)$, sendo as primeiras com pontuação média de 76,91 $\pm 30,27$ e os homens com pontuação média de 57,45 $\pm 22,72$.

Também foi observado que estudantes com sobrepeso ( $72,70 \pm 28,03$ pontos) e obesidade $(96,52 \pm 40,78$ pontos $)$ foram significativamente $(p<0,016)$ mais insatisfeitos que aqueles com IMC baixo $(57,97 \pm 28,45)$ e aqueles com IMC normal $(67,03 \pm 27,46)$.

Analisando a tabela 1, pode-se observar que $10,1 \%$ dos universitários manifestaram algum nível de insatisfação corporal pelo BSQ e, desses, nenhum jovem foi classificado com grave insatisfação (pontuação maior que 167 pontos). Os estudantes da área de humanas $(11,7 \%)$ tiveram a maior frequência na classificação de insatisfação maior que os estudantes da saúde $(11,2 \%)$ e exatas $(6,6 \%)$, diferença não significativa. Já entre os sexos, observou-se que as mulheres foram significativamente classificadas como insatisfeitas em relação aos homens $(p<0,05)$. Entre os grupos de classificação de estado nutricional, também foi observado que os universitários com sobrepeso e obesidade foram significativamente classificados como insatisfeitos em relação àqueles com IMC normal e baixo $(p<0,05)$.

Porém, pela avaliação da escala de silhuetas, conforme pode ser observado na tabela 1, a maioria dos indivíduos foram classificados como insatisfeitos (76,6\%). Desse total, $265(64,63 \%)$ desejaram aumentar sua silhueta atual e 145

Tabela 1. Classificação dos níveis de insatisfação do BSQ e de satisfação corporal da escala de silhuetas em relação às áreas de conhecimento, sexo e EN (Juiz de Fora, MG - 2011)

\begin{tabular}{|c|c|c|c|c|c|c|c|c|}
\hline \multirow[t]{2}{*}{ Variáveis } & \multicolumn{4}{|c|}{ Classificação BSQ } & \multicolumn{4}{|c|}{ Silhuetas } \\
\hline & Livre & Leve & Moderada & Grave & $p$ & Satisfeito & Insatisfeito & $\mathrm{p}$ \\
\hline \multicolumn{9}{|l|}{ Áreas - $n(\%)$} \\
\hline Exatas & $157(93,5)$ & $7(4,2)$ & $4(2,4)$ & - & & $36(21,4)$ & $132(78,6)$ & 0,293 \\
\hline Humanas & $165(88,2)$ & $15(8,0)$ & $7(3,7)$ & - & 0,248 & $40(21,4)$ & $147(78,6)$ & \\
\hline Saúde & $159(88,3)$ & $16(8,4)$ & $5(2,8)$ & - & & $38(21,1)$ & $142(78,9)$ & \\
\hline \multicolumn{9}{|l|}{ Sexo - n (\%) } \\
\hline Masculino & $235(95,9)$ & $6(2,4)$ & $4(1,6)$ & - & $0,000^{\mathrm{a}}$ & $64(22,1)$ & $226(77,9)$ & 0,441 \\
\hline Feminino & $246(84,8)$ & $32(11,0)$ & $12(4,1)$ & - & & $61(24,9)$ & $184(75,1)$ & \\
\hline \multicolumn{9}{|l|}{$\mathrm{EN}-\mathrm{n}(\%)$} \\
\hline Baixo IMC & $45(91,8)$ & $3(6,1)$ & $1(2)$ & - & $0,005^{b}$ & $14(28,6)$ & $35(71,4)$ & 0,183 \\
\hline Normal IMC & $355(91,5)$ & $25(6,4)$ & $8(2,1)$ & - & & $96(24,7)$ & $292(75,3)$ & \\
\hline Sobrepeso & $71(87,7)$ & $6(7,4)$ & $4(4,9)$ & - & & $13(16)$ & $68(84)$ & \\
\hline Obesidade & $10(58,8)$ & $4(23,5)$ & $3(17,6)$ & - & & $2(11,8)$ & $15(88,2)$ & \\
\hline Total & $481(89,9)$ & $38(7,1)$ & $16(3,0)$ & - & & $125(23,4)$ & $410(76,6)$ & \\
\hline
\end{tabular}

EN: estado nutricional; n: número de sujeitos; IMC: índice de massa corporal; \%: porcentagem; p: significância $(<0,05)$.

a: variação significativa de sujeitos mais insatisfeitos do sexo feminino pelo Teste Exato de Fisher $(p=0,000)$ e também pelo teste qui-quadrado de associação linear de Pearson $(p=0,000)$.

b: variação significativa entre a classificação do estado nutricional e a classificação do BSQ pelo Teste Exato de Fisher $(p=0,005)$ e também pelo teste qui-quadrado de associação linear de Pearson ( $p=0,001)$. 
$(35,36 \%)$ queriam diminuí-la. No geral, os homens manifestaram o desejo de uma silhueta maior que a atual (39,9\%), já entre as mulheres, a maioria desejou obter uma silhueta menor que a atual $(61,7 \%)$.

Ainda em relação ao EN, vale ressaltar que aproximadamente $20,0 \%$ das mulheres com baixo e normal IMC foram classificadas com leve e moderada insatisfação corporal pelo BSQ. Já entre os homens, juntando aqueles com baixo IMC, normal IMC e sobrepeso, o percentual de jovens classificados como insatisfeitos não chegou a 5\%. O gráfico 1 mostrou que os universitários com o IMC mais elevado apresentaram maiores índices de insatisfação corporal em ambos os sexos. No entanto, foi perceptível que em todas as classificações do EN as mulheres tiveram níveis de insatisfação mais elevados em relação aos homens.

Por meio do modelo de regressão logística, verificou-se que os jovens com sobrepeso e obesidade tiveram maiores chances de serem mais insatisfeitos com sua imagem corporal (OR: 3,174; $p=0,000$ ) que aqueles com baixo e normal IMC. Já as mulheres (OR: 1,597; p: 0,164) e as áreas de conhecimento foram fatores de risco sem significância (Tabela 2) para com os níveis de insatisfação.

Tabela 2. Modelo de regressão logística binária para a classificação de satisfação corporal da Escala de Silhueta em relação a sexo, peso, estatura, IMC, EN e às áreas de conhecimento (Juiz de Fora, MG - 2011)

\begin{tabular}{lcccccc}
\hline $\begin{array}{c}\text { Variáveis } \\
\text { explicativas }\end{array}$ & $\boldsymbol{\beta}$ & EP & $\begin{array}{c}\text { Estatística } \\
\text { Wald }\end{array}$ & GL & $\boldsymbol{P}$ & OR \\
\hline Meninas & 0,468 & 0,336 & 1,94 & 1 & 0,437 & 1,424 \\
$\begin{array}{l}\text { Sobrepeso/ } \\
\text { Obesidade }\end{array}$ & 0,353 & 0,454 & 0,605 & 1 & $0,000^{\mathrm{a}}$ & 3,174 \\
Saúde & - & - & 1,967 & 2 & 0,374 & - \\
Humanas & 0,280 & 0,257 & 1,185 & 1 & 0,276 & 0,756 \\
Exatas & 0,057 & 0,275 & 0,042 & 1 & 0,837 & 1,058 \\
\hline
\end{tabular}

EN: estado nutricional; EP: erro-padrão; GL: grau de liberdade; p: significância; OR: odds ratio.

a: valores significativos $(p<0,05)$.

\section{DISCUSSÃO}

De acordo com os achados da literatura, são escassos os estudos que investigaram a insatisfação com a imagem corporal em diferentes áreas de conhecimento, utilizando instrumentos adaptação transcultural para uma população específica ${ }^{7,12}$

A média total de pontos do BSQ foi de $68,00 \pm 28,74$, os homens apresentaram média de pontos de 57,45 $\pm 22,72$ e as mulheres apresentaram média de 79,61 \pm 30,27 pontos. No estudo de validação do BSQ com estudantes de medicina da cidade de São Paulo, Brasil, Di Pietro e Silveira ${ }^{14}$ verificaram que as médias de pontuação tanto de homens $(58,7 \pm$ $25,1)$ quanto de mulheres $(89,7 \pm 31,3)$ estavam acima dos resultados encontrados. Em relação à classificação do BSQ, verificou-se que a maior parte da amostra $(89,9 \%)$ esteve livre de insatisfação corporal e, também, nenhum jovem foi classificado com grave insatisfação corporal, ou seja, eles tiveram pontuação maior que 167 pontos no BSQ. Estudos semelhantes encontraram $59,6 \%$ e $82,9 \%$ de universitárias de nutrição e educação física livres de insatisfação, respectivamente ${ }^{10,11}$.

No presente estudo, não foram encontradas variações significativas nas médias gerais de insatisfação entre os estudantes das diferentes áreas de conhecimento, porém pode-se observar que os estudantes da área de saúde e humanas, sem significância, tiveram maiores índices e frequência de classificação de insatisfação corporal que os alunos a área de exatas. Uma explicação para isso pode ser o fato de ter havido maior número de mulheres pesquisadas nas áreas de saúde e humanas em relação à área de exatas: 59,45\% saúde, $54,55 \%$ humanas e $48,22 \%$ exatas.

Esse resultado é diferente de pesquisas que encontraram maior insatisfação corporal em jovens dos cursos da área da saúde, pelo fato de esses jovens tratarem de diversas questões vinculadas ao corpo humano 7,8 . Cursos como educação física ${ }^{11}$, nutriçãa ${ }^{10}$, psicologia ${ }^{8}$ e enfermagem apresentaram maior prevalência de insatisfação corporal. Para Bosi et al.10, 40,4\% de estudantes de nutrição manifestaram insatisfação com sua imagem corporal. Também Moreira et al. ${ }^{20}$ encontraram que $50 \%$ das estudantes de medicina na Bahia apresentaram algum grau de insatisfação corporal.

Foi observado que as mulheres e todos aqueles que estavam com IMC acima do normal tiveram maiores médias de insatisfação corporal, a partir do BSQ. Isso já era esperado, porque resultados semelhantes já foram encontrados na literatura ${ }^{7,10-12}$. No estudo de Quadros et al. ${ }^{12}$, por exemplo, em avaliação da IC em universitários e sua relação com o estado nutricional e o sexo, concluiu-se que as mulheres e os indivíduos com excesso de peso também apresentaram maior insatisfação. Porém, foi interessante observar que 20\% das mulheres com baixo e normal IMC apresentaram insatisfação corporal leve e moderada. McCabe e Ricciardelli21 ressaltaram que muitas mulheres com IMC adequado ou baixo manifestam insatisfação corporal por causa de uma distorção com a própria imagem. Também Alvarenga et al. ${ }^{7}$ mostraram que estudantes eutróficas também apresentaram desejo em ter uma silhueta menor. Isso evidencia que as mulheres por si só, seja por influência do meio em que vive ou da cultura midiática, apresentam insatisfação corporal maior que os homens, mesmo levando em questão o estado nutricional. Esse fato pode ser observado no gráfico 1, no qual em todas as classificações do estado nutricional as mulheres tiveram maiores índices de insatisfação corporal.

A partir dos resultados encontrados nesta pesquisa, percebeu-se que apenas 10,1\% dos universitários foram classificados como insatisfeitos com sua IC, no entanto $76,6 \%$ manifestaram o desejo de possuir uma silhueta diferente 
da selecionada atualmente. Dessa forma, enquanto o BSQ aponta que a maior parte dos indivíduos está livre de insatisfação, a escala de silhueta mostra o oposto. Apesar de se tratar do mesmo componente da IC, o primeiro instrumento avalia pensamentos, sentimentos e comportamentos do indivíduo em relação ao seu corpo; já o segundo, apenas o tamanho e a forma corporal.

Gardner e Brown ${ }^{22}$ compararam metodologias de avaliação da insatisfação com a IC e concluíram que a Escala de Silhuetas pode superestimar os valores encontrados. Isso pode explicar em parte a expressiva diferença encontrada em nossa amostra. Além disso, esse método limita as opções de escolha do indivíduo, tendo em vista que ele deve se enquadrar em uma das figuras existentes, não podendo escolher um meio termo, por isso a necessidade de utilizar mais de um instrumento para avaliar com mais respaldo a insatisfação corporal de determinada população. Porém, é um instrumento validado para essa população específica, permitindo a confiabilidade do estudo ${ }^{23}$.

As figuras mais apontadas como ideais por ambos os sexos no estudo foram aquelas referentes ao IMC considerado normal pela OMS (18 a $\left.25 \mathrm{~kg} / \mathrm{m}^{2}\right)^{18}$. Esse dado é positivo, pois sugere que os indivíduos desejam ter um corpo dentro dos valores considerados adequados pela $\mathrm{WHO}^{19}$.

Na tentativa de verificar qual variável melhor prediz o nível de satisfação a partir da Escala de Silhuetas, foi realizada uma regressão logística binária, também com a classificação do BSQ. Essa análise apontou as variáveis peso corporal, estatura e IMC como fatores de risco para o desenvolvimento da insatisfação corporal. Como o peso corporal e a estatura compõem o IMC, podemos considerar este como o principal preditor da insatisfação corporal. A relação entre insatisfação e IMC já foi amplamente estudada na literatura, e as pesquisas apontam para uma relação direta entre as variáveis, ou seja, quanto maior o IMC, maior a insatisfação ${ }^{8,12}$.

Um dos fatores limitantes do presente estudo foi o fato de analisar de maneira transversal um constructo inconstante e influenciado por diversos fatores. No entanto, acredita-se que esse delineamento foi satisfatório para abordar o problema do presente estudo, ao estimar a frequência da manifestação da insatisfação corporal nos estudantes de uma universidade brasileira. Outro fator limitante a ser considerado foi que outros marcadores de avaliação do tecido adiposo poderiam ser utilizados para serem realizadas outras associações com os índices de insatisfação corporal, além do IMC, que não ressalta a diferença de tecido adiposo e massa magra. A avaliação do peso corporal e da estatura autorreferidas pode ter trazido algumas interferências nos achados, subestimando ou superestimando o IMC dos universitários ${ }^{24}$. Contudo, estudos recentes têm utilizado extensivamente esse procedimento para avaliação do estado nutricional e indicam a validade desse método em estudos populacionais para fornecer informações confiáveis do estado nutricional da população adulta brasileira ${ }^{25,26}$.

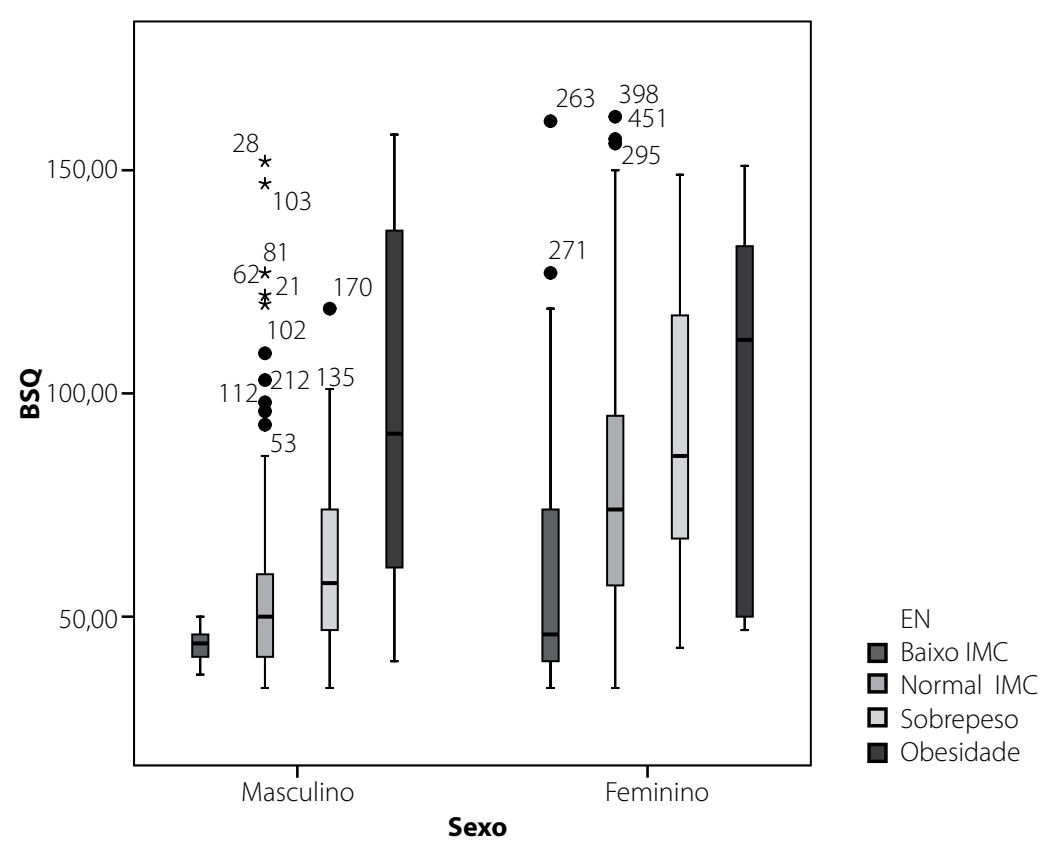

Gráfico 1. Pontuação do BSQ em relação à classificação do estado nutricional entre os sexos. EN: estado nutricional; BSQ: Body Shape Questionnaire. 
Outro fator possivelmente limitante foi a forma bidimensional de apresentação da Escala de Silhuetas, podendo implicar falhas na representação total do corpo e na distribuição da gordura, afetando a formação individual da IC. Entretanto, a utilização desse instrumento se justifica pela facilidade e praticidade para aplicação, pela boa fidedignidade e frequente utilização em estudos epidemiológicos com a população universitária $24,27$.

Observou-se o quanto foi importante analisar a insatisfação corporal nas diferentes áreas de conhecimento, considerando o fato de que muitas pesquisas ressaltaram que o ambiente sociocultural $\left.\right|^{3,4,8,12,27}$ e de estudo do indivíduo pode interferir em sua IC. Por meio dessa avaliação, pode-se perceber que alguns fatores como o estado nutricional e o sexo são de extrema importância na avaliação de algum componente da imagem corporal de jovens universitários de diferentes áreas de conhecimento.

\section{CONCLUSÃO}

Concluiu-se nesta pesquisa que a maioria dos jovens esteve livre de insatisfação corporal, porém a grande maioria manifestou o desejo de ter uma silhueta diferente da atual. Em relação às áreas de conhecimento, percebeu-se que não houve diferença nos níveis de insatisfação, porém o sexo e o EN foram fatores determinantes para maior prevalência de insatisfação corporal dos universitários pesquisados.

Outras variáveis comportamentais relacionadas à IC que não foram controladas neste estudo podem ser consideradas em futuras investigações, tais como: nível socioeconômico, nível de atividade física, influência da mídia, amigos e familiares, depressão, autoestima, comportamento alimentar e uso de esteroides anabolizantes. O conhecimento amplo das variáveis que influenciam a insatisfação corporal é importante para conscientizar os jovens a terem uma relação positiva com sua forma física. Assim, profissionais da saúde vinculados às questões relacionadas à aparência corporal poderão orientar os jovens a tomarem atitudes saudáveis que possam deixá-los mais satisfeitos com sua imagem corporal.

\section{AGRADECIMENTOS}

A todos participantes, pela disponibilidade e incentivo para que os alunos participassem desta pesquisa.

\section{CONFLITOS DE INTERESSE}

Os autores declararam não haver conflitos de interesse.

\section{REFERÊNCIAS}

1. Slade PD. What is body image? Behav Res Ther. 1994;32(5):497-502.

2. Garner DM, Garfinkel PE. Body Image in anorexia nervosa: measurement theory and clinical implications. Int J Psychiatry Med. 1981;11(3):263-84.

3. Van den Berg P, Thompson JK, Obremski-Brandon K, Coovert M. The tripartite influence model of body image and eating disturbance: a covariance structure modeling investigation testing the meditational role of appearance comparison. J Psychosom Res. 2002:53:1007-29

4. Adami F, Frainer DES, Santos JS, Fernandes TC, De-Oliveira FR. Insatisfação corporal e atividade física em adolescentes da região continental de Florianópolis. Psicol Teor Pesqui. 2008;24(2):143-9

5. Timerman F, Scagliusi FB, Cordás TA. Acompanhamento da evolução dos distúrbios de imagem corporal em pacientes com bulimia nervosa, ao longo do tratamento multiprofissional. Rev Psiq Clín. 2010;37(3):113-7.

6. Vieira VCR, Priore SE, Ribeiro SMR, Franceschini SCC, Almeida LP. Perfil socioeconômico, nutricional e de saúde de adolescentes recém-ingressos em uma universidade pública brasileira. Rev Nutr. 2002;15(3):273-82

7. Alvarenga MS, Philippi ST, Lourenço BH, Sato PM, Scagliusi FB. Insatisfação com a imagem corporal em universitárias brasileiras. J Bras Psiquiatr. 2010;59(1):44-51.

8. Costa LCF, Vasconcelos FAG. Influência de fatores socioeconômicos, comportamentais e nutricionais na insatisfação com a imagem corporal de universitárias em Florianópolis, SC. Rev Bras Epidemiol. 2010;13(4):665-76

9. Bosi MLM, Uchimura KY, Luiz RR. Eating behavior and body image among psychology students. J Bras Psiquiatr. 2009;58(3):150-5.

10. Bosi MLM, Luiz RR, Morgado CMC, Costa MLS, Carvalho RJ. Autopercepção da imagem corporal entre estudantes de nutrição: um estudo no município do Rio de Janeiro. J Bras Psiquiatr. 2006;55(2):108-13.

11. Bosi MLM, Luiz RR, Uchimura KY, Oliveira FP. Comportamento alimentar e imagem corporal entre estudantes de educação física. J Bras Psiquiatr. 2008;57(1):28-33.

12. Quadros TMB, Gordia AP, Martins CR, Silva DAS, Ferrari EP, Petroski EL. Imagem corporal em universitários: associação com estado nutricional e sexo. Motriz. 2010;16(1):78-85.

13. Thomas JR, Nelson JK, Silverman SJ. Métodos de pesquisa em atividade física. São Paulo: Artmed; 2007.

14. Di Pietro M, Silveira DX. Internal validity, dimensionality and performance of the Body Shape Questionnaire in a group of Brazilian college students. Rev Bras Psiquiatr. 2009;31(1):21-4.

15. Universidade Federal de Juiz de Fora. Disponível em: http://www.uff.br. Acesso em: Set 20,2009

16. Cooper PJ, Taylor MJ, Cooper Z, Fairbum CG. The development and validation of the Body Shape Questionnaire. Int J Eat Disord. 1987;6(4):485-94.

17. Kakeshita IS, Silva AIP, Zanata DP, Almeida SS. Construção e fidedignidade teste-reteste de escalas de silhuetas brasileiras para adultos e crianças. Psicol Teor Pesqui. 2009;25(2):263-70.

18. Kakeshita IS, Almeida SS. Estudo da relação entre o índice de massa corporal e o comportamento alimentar de adultos. Nutrire Rev Soc Bras Aliment Nutr. 2008;33(1):21-30.

19. World Health Organization. WHO Expert Committee on Physical Status: The use and interpretation of anthropometry physical status. Geneva: World Health Organization; 1995 (WHO Technical Report Series, v. 854).

20. Moreira LAC, Azevedo ABG, Queiroz D, Moura L, Santo DE, Cruz R, et al. Body image in a sample of undergraduate medical students from Salvador, Bahia, Brazil. J Bras Psiquiatr. 2005:54(4):295-7.

21. McCabe MP, Ricciardelli LA. A longitudinal study of pubertal timing and extreme body change behaviors among adolescent boys and girls. Adolescence. 2004;39:145-66. 
22. Gardner RM, Brown DL. Comparison of video distortion and figural drawing scale for measuring and predicting body image dissatisfaction and distortion. Pers Indiv Differ. 2010;49(7):794-8.

23. Thompson JK. The (mis)measurement of body image: ten strategies to improve assessment for applied and research purposes. Body Image. 2004;1:7-14.

24. Coqueiro RS, Petroski EL, Pelegrini A, Barbosa AR. Insatisfação com a imagem corporal: avaliação comparativa da associação com estado nutricional em universitários. Rev Psiquiatr Rio Gd Sul. 2008;30(1):31-8.
25. Oliveira AGP, Oliveira TP, Ferreira MEC, Lima JRP. Diferença entre peso e estatura autorreferidos e aferidos para o cálculo do índice de massa corporal e sua relação com a imagem corporal de mulheres de academia de ginástica. HU Rev. 2008;34(3):179-83.

26. Brunt AR, Rhee YS. Obesity and lifestyle in U.S. college students related to living arrangements. Appetite. 2008;51:615-21.

27. Kakeshita IS, Almeida SS. Relação entre índice de massa corporal e a percepção da autoimagem em universitários. Rev Saude Publica. 2006;40(3):497-504. 